\title{
Modeling and Fabrication of an RF MEMS Variable Capacitor with a Fractal Geometry
}

\author{
A. M. Elshurafa and K. N. Salama \\ Electrical Engineering \\ King Abdullah University of Science \& Technology (KAUST) \\ Thuwal, Saudi Arabia \\ elshurafa@ieee.org, khaled_salama@ieee.org
}

\author{
P. H. Ho \\ ASM Pacific Technology Hong Kong Limited \\ Kwai Chung, Hong Kong \\ pakhung.ho@kaust.edu.sa
}

\begin{abstract}
In this paper, we model, fabricate, and measure an electrostatically actuated MEMS variable capacitor that utilizes a fractal geometry and serpentine-like suspension arms. Explicitly, a variable capacitor that possesses a top suspended plate with a specific fractal geometry and also possesses a bottom fixed plate complementary in shape to the top plate has been fabricated in the PolyMUMPS process. An important benefit that was achieved from using the fractal geometry in designing the MEMS variable capacitor is increasing the tuning range of the variable capacitor beyond the typical ratio of 1.5 . The modeling was carried out using the commercially available finite element software COMSOL to predict both the tuning range and pull-in voltage. Measurement results show that the tuning range is 2.5 at a maximum actuation voltage of $10 \mathrm{~V}$.
\end{abstract}

\section{INTRODUCTION}

The most recent $4 \mathrm{G}$ devices are designed with specific emphasis on the data traffic challenge; because there is an increasing number of bands, all the circuits and devices related to the design of wireless systems need to be enhanced including the power amplifiers, filters, and antennas. One of the very promising ways to enable these enhancements is to use tunable RF devices based on microelectromechanical systems (MEMS) technology [1], and RF MEMS variable capacitance devices in general (i.e. switches, switchedcapacitors, and analog varactors), pose themselves as very strong candidates for this purpose because they exhibit some favorable characteristics including low loss $(<0.2 \mathrm{db})$, high power handling capability (1-10 Watts), virtually nonexistent power consumption and leakage, linearity, and ability to operate at frequencies greater than $100 \mathrm{GHz}$ [2-3].

Despite the above-mentioned advantages, electrostaticallyactuated analog MEMS variable capacitors (MVC) in specific do suffer from the pull-in effect which limits the theoretical tuning range $(T R)$ in the parallel-plate type MVC to 1.5 [4]. Many successful attempts to overcome this pull-in have been reported (see $[5,6]$ for example). In this paper, we design an MVC that utilizes a fractal geometry to overcome the pull-in limitation and to eliminate also the need of creating etching holes within the structure that are required for releasing the sacrificial oxide [7]. In addition to utilizing fractal geometries
[8], the design also purposefully uses the warping caused due to the residual stress and the concept of zipping advantageously.

\section{PROPOSED VARIABLE CAPACITOR}

Fig. 1 shows a 3D isometric view of the MVC created using SEMulator3D (www.coventor.com), while keeping in mind that it is intended to manufacture this device in the PolyMUMPS process (www.memscap.com). As such, the top plate will be created using the Poly2+Metal layers, whereas the bottom plate will be created using the Poly0 layer.

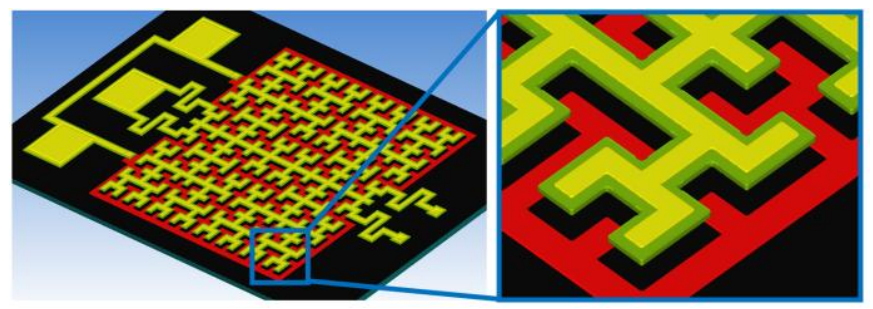

Fig. 1. A 3D isometric view of the MVC, where the complete structure with serpentine suspension arms and measurement pads are shown.

By taking a close look at Fig. 1, we note that the top plate is complementary in shape to the bottom plate, i.e. nowhere does the bottom plate exist under the top plate and nowhere does the top plate exist under the bottom plate. Further, the capacitance is solely generated due to fringing fields. When applying a DC voltage, the top plate is actuated towards the bottom via the resultant electrostatic force. Given that both plates are complementary to each other in shape, no matter how high the actuation voltage reaches, we do not worry about the plates touching each other and causing a short; the maximum that would happen is for the top plate to fill in the void present within the bottom plate, which is similar to how jigsaw puzzle pieces are joined. Nonetheless, we intentionally create the structure with a horizontal separation between the top and bottom plates of $2 \mu \mathrm{m}$ to account for any fabrication imperfections that may occur and consequently cause shorting. Note also that the geometry of the fractal MVC 
follows the $5^{\text {th }}$ order Moore's iteration, and the reason behind using the Moore's fractal was detailed in [9].

\section{COMSOL CAPACITANCE AND DEFLECTION SIMULATIONS}

\section{A. Capacitance Simulation}

Extracting the capacitance and the pull-in voltages in the well-known parallel-plate varactors is relatively easy and the expressions available are able to anticipate the values rather well. However, it is difficult to deduce closed-form expressions for the case of this paper and hence we resort to a numerical solution using the finite element software package COMSOL (www.comsol.com).

The objective of this simulation is to try and anticipate, approximately, the attainable $T R$ from using the Moore's fractal. With that in mind, and due to symmetry, we create one-quarter of the Moore's fractal in COMSOL to evaluate the overall capacitance, and vary the altitude of the top plate from the substrate.

Before attempting to anticipate the performance of the capacitor through simulation, it is important to account for warping that will occur due to residual stresses [4]. This warping will cause the initial capacitance to decrease and hence, the tuning range will increase. By taking a close look at our structure in Fig. 1, it could be easily deduced that the outer parts of the capacitor will warp upwards and will undergo more warping when compared to the middle parts of the capacitor.

We perform the simulation to extract the tuning range, keeping in mind the warping that will take place, after considering the following two simplifying assumptions. First, and despite that the distance between Poly2 and Poly0 should be $1.2 \mu \mathrm{m}$ in PolyMUMPS (which is the combined thickness of Oxide1 and Oxide 2 after being etched with the via step), we will assume that the distance separating the two plates is $5 \mu \mathrm{m}$. The separation of $5 \mu \mathrm{m}$ represents an average distance that accounts for the overall warping of the plate that will take place due to residual stresses. This assumption was made because it is difficult to create the top plate in 3D with a warped shape. Note that a distance other than $5 \mu \mathrm{m}$ could have been chosen, but the distance of $5 \mu \mathrm{m}$ represents a reasonable average for the overall warping that will take place (more details will follow in the measurement section). Further, and from the plot that will be generated, it is immediately possible to extract the tuning range for lower separating distances.

The second assumption is to ignore the parasitic capacitance that will be contributed from the measurement pads because detailed analysis regarding the pads in PolyMUMPS is available [10]. Explicitly, the pads will contribute a constant capacitance of approximately $0.2 \mathrm{pF}$.

With the aforementioned assumptions in mind, the structure was created in COMSOL as shown in Fig. 2, and electrostatic-parametric analysis was performed by varying the altitude from $5 \mu \mathrm{m}$ all the way down to $0 \mu \mathrm{m}$, in which the latter case represents the top plate resting on the nitride. We also include a thin layer under the nitride to account for the parasitics contributed from the substrate. The extracted capacitance is plotted versus the altitude, and it was concluded that the structure would provide a $T R$ of 6.5 as shown in Fig. 3. Note that if we assume that the top plate would be residing at a distance higher than $5 \mu \mathrm{m}$ initially, then the $T R$ would be higher than 6.5. Similarly, if we choose an initial separating distance of $4 \mu \mathrm{m}$ between the top and bottom plates the $T R$ will be lower (Fig. 3 indicates that the $T R$ will be 3 for an initial separation of $4 \mu \mathrm{m}$ ). If we incorporate the measurement pads parasitics, then the $T R$ drops from 6.5 to approximately 2 .

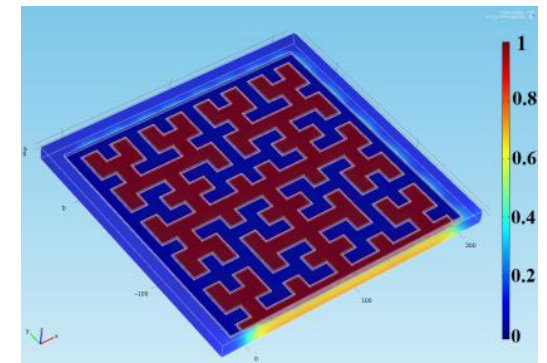

Fig. 2. An electrostatic simulation performed to extract the capacitance. The color bar represents the normalized potential.

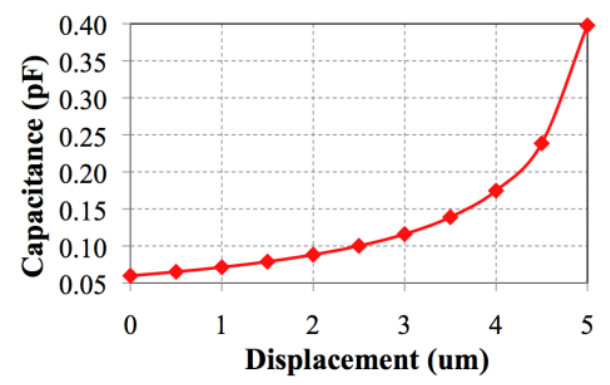

Fig. 3. The simulated $T R$ of a quarter fractal MVC. The figure corresponds to a $T R$ of approximately 6.5 .

\section{B. Deflection Simulation}

The previous section dealt with capacitance without paying any attention to the DC voltage required to actuate the top plate. In this section, we model, once again, one-quarter of the plate and try to estimate the voltage required for the top plate to travel the distance that separates it from the substrate. As explained earlier, the middle section of the plate will undergo the least amount of residual-stress warping, while the outer parts of the top plate will suffer the most warping. Here, we are interested in the voltage that will result in a total deflection of $1.2 \mu \mathrm{m}$ anywhere in the top plate irrespective of its position within the plate itself, because it is at this distance that the pull-in will occur and thus the top plate will come in contact with the nitride (we also know that this point is surely within the vicinity of the middle parts of the plate).

Once there is contact between the top plate and nitride, zipping will take place and will eventually result in the total plate becoming in full contact with the nitride. In this section, it is intended to find the voltage at which the collapse will 
happen since a theoretical solution would be difficult to find given that the capacitances present are fringing capacitances with no closed-form expressions available to evaluate them. In order to do so, an assumption that will reduce the complexity of the simulation and reduce the computation required, without sacrificing much of the accuracy, will be made. The assumption is illustrated in the following.

The thickness of the nitride layer, which separates the plates of the varactor from the substrate, is $0.6 \mu \mathrm{m}$. Immediately under this nitride layer, a very thin portion of the substrate is heavily doped with phosphorus [11], and as such the parasitic capacitances from the substrate cannot be ignored [12]. As such, the substrate will emulate the role of a virtual bottom plate that is $1.2 \mu \mathrm{m}+0.6 \mu \mathrm{m}=1.8 \mu \mathrm{m}$ away from the top plate.

When the dielectric layer separating two plates is air only, then the pull-in occurs at exactly one-third of the initial separating distance. For the cases of this paper, where the substrate is separated from the top plate by air and nitride, then the new pull-in distance, $g_{\text {new-pull-in }}$, is [12]:

$$
g_{\text {new-pull-in }}=\left(g_{0}+R t\right) / 3
$$

where $g_{0}$ is the initial separating distance between the top plate and the nitride layer (i.e. $1.2 \mu \mathrm{m}), R$ is the ratio of the dielectric constant of air to that of nitride (i.e. $~ 0.1333$ ), and $t$ is the thickness of the nitride layer (i.e. $0.6 \mu \mathrm{m}$ ). Hence, the distance $g_{\text {new-pull-in }}$ is $0.63 \mu \mathrm{m}$ as opposed to $1.8 \mu \mathrm{m} / 3=0.6 \mu \mathrm{m}$, which represents $30 \mathrm{~nm}$ of difference only. As such, and for practical purposes, including the insulator layer in analysis will not have an effect that justifies that added complexity because the condition $g_{0} \gg R t$ is usually satisfied in MEMS. With the previous analysis put forward, it is safe to carry out the simulations for detecting pull-in ignoring the nitride layer.

A multi-physics simulation was performed. Explicitly, we use the solid mechanics physics, electrostatic physics, and the moving mesh physics in COMSOL. Normally, any finite element software determines and solves the differential equations of fixed meshes (reference frame). By adding a moving-mesh physics to the problem, the software automatically transforms the equations and uses them to calculate results in the reference and moving frames [13]. Then a parametric study was done in order to arrive at a deflection of $0.6 \mu \mathrm{m}$. One drawback about this mutliphysics simulation is the significant memory and processing time required to obtain a solution. This problem is further exacerbated given that the aspect ratios in MEMS are usually very high which results in an increased number of elements in the mesh. Further, and because pull-in represents a mathematical instability, finding the pull-in voltage requires some trial and error to arrive at the magnitude at which the solution does not converge. Moreover, the closer the solution is to the pull-in voltage, the longer the time required for the solution to converge. The mathematical instability that the pull-in represents translates experimentally to the top plate snapping onto the bottom plate.
Fig. 4 shows the simulation result for the top plate with a deflection of $0.6 \mu \mathrm{m}$ approximately, which was acquired at an actuation voltage of $5 \mathrm{~V}$. As can be seen, the points that are further from the suspension arms deflect the most as expected. The total mesh consisted of approximately 170,000 elements, approximately 1,000,000 degrees of freedom, and the solution time required to calculate the deflection for a single voltage value was nearly 30 minutes, on a T7500 Dell workstation possessing two Xeon X5550 processors (each of which containing four processers for a grand total RAM of 24GB), which indicates that the solution is indeed computationally-intense.

Before leaving this section, we alert the reader that the finite element method in itself is an approximation. A finer mesh could have been used at the cost of a considerably longer solution time. However, and for proof-of-concept purposes, we can conclude from the above simplified simulations, with acceptable degrees of confidence that: 1) the $T R$ range of the fractal MVC is higher than the conventional limit of 1.5 , and 2) the voltage that will cause the middle part of the top plate to collide with the substrate will be in the vicinity of $5 \mathrm{~V}$.

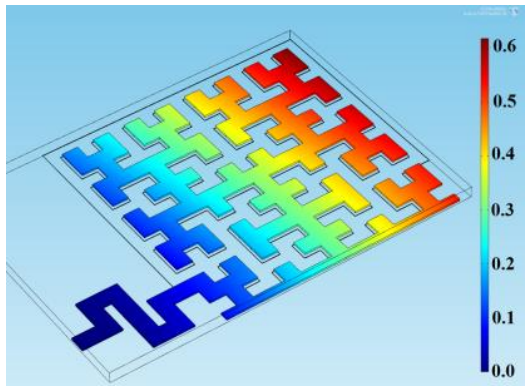

Fig. 4. A multi-physics simulation of one-quarter of the variable capacitor showing that the displacement is maximum at the outer parts of the plates. The actuation voltage used was $5 \mathrm{~V}$.

IV. MEASUREMENT Results

Fig. 5 shows a scanning electron microscope (SEM) image of the fabricated MVC in the PolyMUMPS process. We also include an optical profiler image in Fig. 6 for the capacitor to quantify experimentally the severity of the warping present. It was found that the outer parts of the capacitor are approximately $9 \mu \mathrm{m}$ away from the nitride, which makes our assumption for choosing a distance of $5 \mu \mathrm{m}$ for simulation purposes in Section III a reasonable one.

The tuning behavior of the MVC is shown in Fig. 7, and corresponds to a tuning range of 2.5. For reliability purposes, the actuation was performed twice, and as can be seen, the $\mathrm{CV}$ behavior in both cases is very similar. From the measured tuning range, we can deduce that the simulation assumptions that were made were indeed reasonable. Fig. 7 also shows that the first pull-in occurs at a voltage of approximately $4 \mathrm{~V}$ compared to a simulated value of $5 \mathrm{~V}$. As expected, the tuning behavior is not fully linear due to the parasitics coming from the substrate, which is also the case for all parallel-plate MVCs. Generally however, the linearity and $T R$ herein are enhanced when compared to previously reported parallelplate MVCs [1-3] due to the multiple pull-ins [14], and the 
linearity is particularly better than that reported in [14] given the enhanced suspension design.

The $S_{11}$ measurement of the capacitor is shown from a frequency of $1 \mathrm{GHz}$ up to $4 \mathrm{GHz}$ in Fig. 8, and the quality factor $(Q)$ was almost 4 throughout the frequency band. The low $Q$ is attributed to several factors including for example measurement pad and substrate parasitics, series resistance in the suspension, and the lossy polysilicon bottom plate.

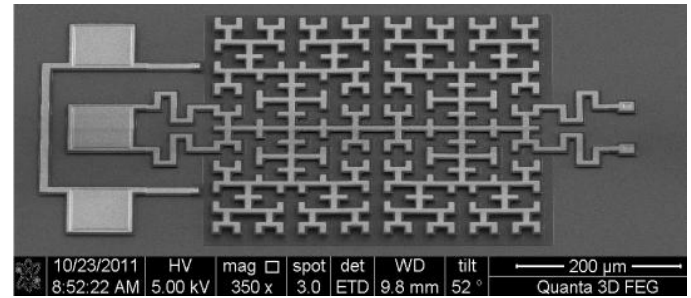

Fig. 5. The proposed MEMS fractal variable capacitor. Notice how the suspension arms in this capacitor are serpentine in shape.

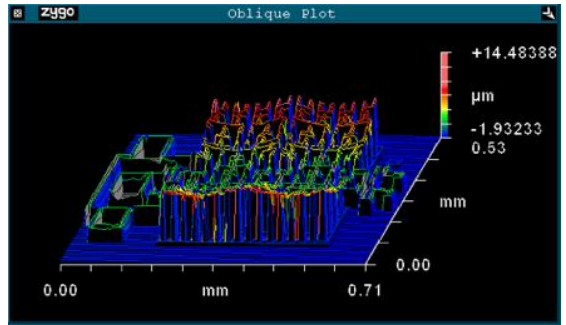

Fig. 6. An optical profiler image for the proposed variable capacitor. It is clear how the outer parts of the top plate undergo more warping than the middle parts of the plate.

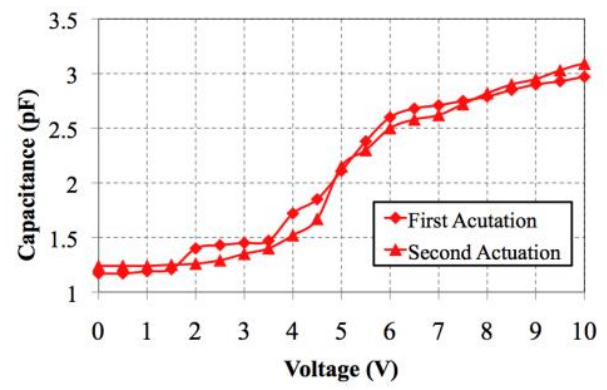

Fig. 7. The measured tuning behavior of the MVC.

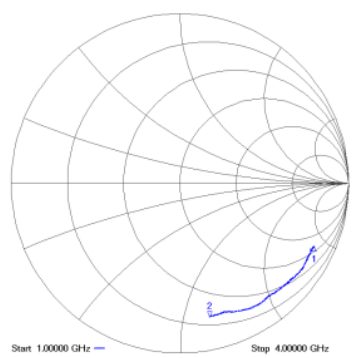

Fig. 8. The $S_{11}$ measurement for the MVC from $1 \mathrm{GHz}$ to $4 \mathrm{GHz}$.

For comprehensiveness, we provide in Fig. 9, two microphotographs of the varactor before and after actuation. In Fig. 9a, the outer parts of the top plate are not as shiny as the middle parts of the capacitor, and these light fringes (shadows) are a result of the warping of the top plate. However, in Fig. 9b, the varactor is shown after actuation (at $10 \mathrm{~V}$ ) and it is clearly observed that the complete plate shows a uniform light intensity, proving that all the parts of the plate are at the same altitude (i.e. resting on the substrate).

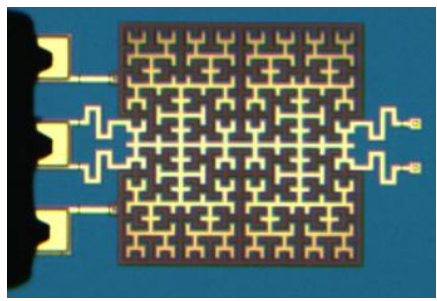

(a)

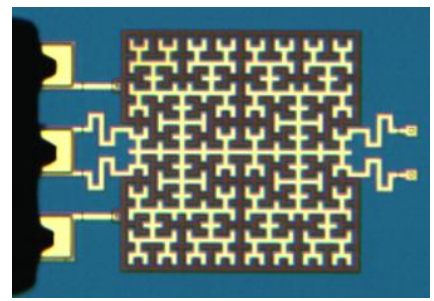

(b)
Fig. 9. A microphotograph of the MVC (a) before and (b) after actuation showing the different light fringes (shadows). See text for details.

\section{CONCLUSION}

We presented a MEMS variable capacitor that can overcome the pull-in limitation by employing fractal geometries. Further, and by carefully assuming realistic and logical assumptions, we showed the time and memory requirement for simulating the problem under consideration could be significantly reduced with little effect on the overall accuracy of the acquired solution.

\section{REFERENCES}

[1] G. M. Rebeiz et al., "Tuning in to RF MEMS," IEEE Microw. Mag., Vol. 10, No. 6, pp. 55-72, 2009.

[2] A. M. Elshurafa, P. H. Ho, and K. N. Salama, "A low voltage RF MEMS variable capacitor with linear $\mathrm{C}-\mathrm{V}$ response," Electron. Lett., Vol. 48, No. 7, pp. 392-393, 2012.

[3] G. M Rebeiz, RF MEMS, Applications, Theory, and Techniques, 2003, Wiley, NY, United States.

[4] A. M. Elshurafa and E. El-Masry, "Design considerations in MEMS parallel plate variable capacitors," IEEE Midwest Symp. Cir. Syst., 2007, pp. 1173-1176.

[5] T. Tsang and M. El-Gamal, "Very wide tuning range microelectromechanical capacitors in the MUMPs process for RF applications," IEEE Symp. VLSI Cir., 2003, pp. 33-36.

[6] C. Han, D. Choi, and J. Boon, "Parallel-plate MEMS variable capacitor with superior linearity and large tuning ratio using a levering structure," IEEE J. Microelectromech. Syst., Vol. 20, No. 6, pp. 1345-1354, 2011.

[7] A. M. Elshurafa and E. El-Masry, "Effects of etching holes on capacitance and tuning range in MEMS parallel plate variable capacitors," IEEE Int'l Workshop Syst. Chip., 2006, pp. 221-224.

[8] A. M. Elshurafa and K. N. Salama, "Two-Layer RF MEMS Fractal Capacitors in PolyMUMPS for S-Band Applications," IET Micro Nano Lett., Vol. 7, No. 5, pp. 419-421, 2012.

[9] A. M. Elshurafa, A. G. Radwan, A. Emira, and K. N. Salama, "RF MEMS fractal capacitos with high self resonant frequencies," IEEE J. Microelectomech. Syst., Vol. 21, No. 1, pp. 10-12, 2012.

[10] A. Dec and K. Suyama, "Micromachined electromechanically tunable capacitors and their applications to RF IC's," IEEE Trans. Microw. Theory Tech., Vol. 46, No. 12, pp. 2587-2596, 1998.

[11] J. Carter, B. Hardy, R. Mahadeven, and S. Wilcenski, "PolyMUMPS design handbook, Rev. 13," MEMSCAP Inc., Bernin, France.

[12] A. M. Elshurafa and E. El-Masry, "MEMS variable capacitance devices utilizing the substrate: II. Zipping varactors," J. Micromech. Microeng., Vol. 20, No. 4, 045028, 2010.

[13] "COMSOL V4 Documentation," COMSOL Inc., Burlington, MA, United States.

[14] A. M. Elshurafa, A. G. Radwan, P. H. Ho, M. H. Ouda, K. N. Salama, "Low voltage puzzle-like fractal MEMS varaible capacitors suppressing pull-in," IET Micro Nano Lett., Vol. 7, No. 9, pp. 965-969, 2012. 